salary for an unskilled worker.

Ivory demand and prices

have reached a point at

which poachers are willing to target well-protected, closely monitored populations. With many poorly protected, softtarget elephant populations now over-harvested (P. Bouche et al. PLoS ONE 6, e20619; 2011), the pressure on the Samburu elephant population may be a harbinger of what is to come for Africa's protected areas. George Wittemyer Colorado State University, Fort Collins, USA. g.wittemyer@colostate.edu David Daballen, Iain DouglasHamilton Save the Elephants, Nairobi, Kenya.

\section{Endangered cultures need protection too}

Many strategies are used to protect wild plants and animals, but we are much more lax when it comes to ancestral cultures that are threatened or endangered worldwide.

We should recognize the few humans still living in isolation as infallible indicators of true wilderness. Both are vanishingly rare and our responsibilities towards them are tremendous. Any country that can boast the presence of an uncontacted group is truly fortunate and should realize that this represents an unusual opportunity to save two irreplaceable assets - a unique people and a wild place

- for the price of one.

Ecuador is one such country. The Tagaeri and the Taromenane clans of the Waorani nation, voluntarily separated from Western civilization in space and time, roam the lowland rainforests of Yasuní, a region that has been documented as housing the greatest species richness on the planet.

In eastern Ecuador, as elsewhere, valuable resources located within indigenous peoples' homelands put them and their cultures at risk. Resorting to violence to push them aside has rightly come to be considered abominable. Financial means (from job offers and donations to promises of improved standards of living) are more acceptable and widely used to pacify native peoples on contact, or simply to get them out of the way - always under the pretext of a country's greater economic good.

A small isolated group of humans with no vote or voice is as valid for preservation as an endangered species. A country such as Ecuador has everything to gain by protecting both intact ecosystems and cultures through hands-off policies that are akin to those used for endangered species.

Kelly Swing San Francisco de Quito University, Quito, Ecuador. kswing@usfq.edu.ec

\section{US Congress right to halt GM salmon}

You are incorrect in assuming that US federal-agency decisions on genetically modified (GM) organisms are always based on sound science and that Congress might be undermining such decisions (Nature 475, 5; 2011).

In the case of GM salmon, the Advisory Committee of the US Food and Drug Administration (FDA) scolded the FDA and the salmon-production company AquaBounty of Waltham, Massachusetts, for overlooking important scientific issues during the approval process. Criticisms included inadequate sample sizes, incomplete data, questionable culling practices, troubling physical abnormalities, a failed test for allergenicity, and poor environmental and scientific assessments. So far, the agency has not corrected any of these oversights.

Furthermore, the FDA has never promulgated mandatory regulations or amended existing ones to cover GM animals. Instead, it announced in its non-binding 2009 Guidance for Industry (see go.nature.com/ nvcrnx) that it would approve such animals under its existing 'new animal' drug law. As a result, the FDA - which has insufficient scientific expertise in assessing fisheries and environmental risk - is giving itself the task of reviewing and assessing GM fish. This is hardly a prescription for sound science.

Rather than blaming

Congress, we should be glad

that it has stepped in to address this serious economic, humanhealth and environmental issue. Andrew Kimbrell, Colin O'Neil Center for Food Safety, Washington DC, USA.

kimbrell@icta.org

\section{Balance the blend of food and fuel}

Biofuels from non-food sources (lignocellulose, for example) are seen as a superior alternative to those from crops such as grain and sugar fuels mistakenly assumed to have caused the 2008 spike in food prices. Competition for farmland continues to stoke the food-versus-biofuel debate, but a more inclusive and less simplistic framework is needed.

In a world where $33 \%$ of the population is overweight, $17 \%$ is undernourished and $40 \%$ of the food in developed countries is wasted, other aspects of food security should be considered. These include access to food (through governance, cost and distribution), nutritional adequacy and consumer behaviour.

Farmers already respond to policy and economic signals such as subsidies and renewable-energy mandates by diversifying their production systems. Integrating food crops, bioenergy feedstock and carbon farming could be an important strategy to counter economic and environmental variability.

Increasing global demand for food and energy means that discussions of land use should include the whole energy sector, not just the minor biofuel component. Other energy industries such as coal-bed methane producers, as well as urbanization, are also competing for arable land and potable water.

Expanding the debate in this way will help to integrate agricultural, energy and natural-resource policies to meet the needs of governments, producers and consumers in achieving food and fuel security, sustainable management of natural resources and reduced greenhouse-gas emissions. Luis C. Rodriguez, Deborah O'Connell CSIRO Ecosystem Sciences, Crace, Australian Capital Territory, Australia. deborah.o'connell@csiro.au

\section{Nanomaterials need} flexible regulation

Andrew Maynard rightly points out that 'one-size-fitsall' definitions are potentially harmful as they cannot capture the range of risks associated with a diversity of nanomaterials (Nature 475, 31; 2011). But in arguing that regulators should instead work with a list of functional attributes to develop proper science-based regulations, he overlooks the fact that knowledge of such attributes, and internationally agreed methods for acquiring it, will take years of research to accumulate.

Given this situation, adopting a functional definition arguably means that, in theory, the most scientifically sound approach to regulation would be to impose a moratorium on the use of nanomaterials until the relevant trigger-point attributes can be established. There would still be the problem of defining the point in the life cycle of a nanoproduct at which such attributes should be measured, given that they would be likely to change with time and context.

The concept of adaptive regulation itself needs to be flexible enough to recognize the limitations of scientific understanding and its legitimacy as a basis for policy.

Fern Wickson Gen Øk Centre for Biosafety, Tromsø, Norway. fern.wickson@genok.org

CONTRIBUTIONS

Correspondence may be sent to correspondence@ nature.com after consulting the author guidelines at go.nature.com/cMCHno. 